\title{
Indifférenciation et singularité dans les courts métrages de Dominique Gonzalez-Foerster
}

François Bovier et Geneviève Loup

\section{(2) OpenEdition}

\section{Journals}

Édition électronique

URL : http://journals.openedition.org/decadrages/514

DOI : $10.4000 /$ decadrages. 514

ISSN : 2297-5977

Éditeur

Association Décadrages

Édition imprimée

Date de publication : 10 octobre 2008

Pagination : 41-51

ISBN : 978-2-9700582-8-1

ISSN : 2235-7823

Référence électronique

François Bovier et Geneviève Loup, «Indifférenciation et singularité dans les courts métrages de Dominique Gonzalez-Foerster », Décadrages [En ligne], 13 | 2008, mis en ligne le 10 octobre 2009, consulté le 03 mai 2019. URL : http://journals.openedition.org/decadrages/514 ; DOI : 10.4000/ decadrages. 514 


\section{Indifférenciation et singularité dans les courts}

\section{métrages de Dominique Gonzalez-Foerster ${ }^{1}$}

\author{
par François Bovier et Geneviève Loup
}

Dans un entretien avec Hans Ulrich Obrist ${ }^{2}$, Dominique GonzalezFoerster envisage sa démarche artisitique à partir de la notion de tropicalisme $^{3}$. Le constat d'un effet très visible de «tropicalisation» $\mathbf{4}$ qui gagne en 2001 la ville de Paris (pluies tropicales, végétation luxuriante, prolifération soudaine d'araignées, etc.), la conduit à associer son travail à un phénomène de désorientation spatiale. La transformation de la végétation renouvelle la perception de l'espace urbain et l'identité d'un site: selon l'artiste, «la modernité en architecture prend toute sa force quand elle est confrontée à un milieu organique lui-même puissant» $\mathbf{5}$. L'enchevêtrement du minéral et du végétal suscite des réminiscences d'un ailleurs exotique, expérience constitutive d'un territoire mental. La tropicalisation provoque un mouvement d'homogénéisation de l'environnement qui se cristallise à travers une progressive indifférenciation des villes, soulignée par la relative perte des repères spatio-temporels dans l'œuvre de Dominique Gonzalez-Foerster.

\section{Tropicalisation}

Les premières interrogations sur les métissages culturels, et en particulier la récupération de signes non indigènes, remontent au mouvement anthropophagiste que Tarsila do Amaral, Mário et Oswald de Andrade animent de 1928 à 1929: le "Manifeste anthropophage», publié par Oswald de Andrade dans la Revue d'anthropophagie 6 , revendique un phénomène d'assimilation critique des idées et des arts européens par la culture brésilienne, signalant ainsi un mécanisme d'appropriation et de refaçonnement de la culture du colon - ce qui implique une inversion de l'hégémonie de l'Occident et une absorption de ses points de repères, à travers un syncrétisme irrévérencieux. Le cinéma novõ brésilien, qui participe au courant tropicaliste, a redéfini une esthétique des marges et de la résistance, notamment à travers le manifeste «Esthétique de la faim» de Glauber Rocha ${ }^{7}$. Bien que le mouvement anthropophagiste et
1 Tous les courts métrages envisagés ici sont issus du DVD Parc Central (MK2, collection Anna Sanders Films, ( 2006). Ils présentent des divergences et des ajouts par rapport aux films et aux pièces vidéographiques qui seront extraits à partir de ces matrices, Dominique Gonzalez-Foerster attribuant à ces repérages et premiers tournages qu'elle assemble en un DVD le statut d'un long métrage inédit.

2 Hans Ulrich Obrist, "Dominique GonzalezFoerster", Conversations. Vol. 1, Editions Manuella, Paris, 2008, pp. 305-320.

3 En 1967, l'artiste Hélio Oiticica expose au Musée d'art moderne de Rio Tropicália, œuvre qu'il désigne comme une forme "pénétrable": cette installation intègre de manière critique les clichés de la tropicalité (sable de plages, oiseaux colorés, paysages luxuriants) tout en les confrontant à un poste de télévision. Le courant tropicaliste, dont Oiticica se distancie rapidement, prône un métissage des références, principalement dans la musique (Caetano Veloso sort en 1968 un album également intitulé Tropicália).

4 Et Hans Ulrich Obrist de préciser aussitôt: "Tu parles de tropicalisation et non de tropicalité... un peu à la manière d'Edouard Glissant qui parle de créolisation et non de créolité." (Id., p. 306).

$5 \mathrm{lbid}$.

6 Oswald de Andrade, Anthropophagies, Flammarion, Paris, 1982, traduit par Jacques Thiériot ["Manifesto antropófago ", Revista de antropogagia, $n^{\circ} 1$, mai 1928].

7 Glauber Rocha, "Culture de la faim - cinéma de la violence" [1965], in Cinéma 67, n 113, février 1967, repris dans René Gardies, Glauber Rocha, Seghers, Paris, 1974, pp. 128-129. 
le cinéma novõ ne soient pas explicitement convoqués par Dominique Gonzalez-Foerster et Hans Ulrich Obrist, les films et les installations de l'artiste prolongent la question des références culturelles dans un contexte postcolonial qui nécessite un repositionnement de la part des Occidentaux. Dès les premières décennies du $\mathrm{XX}^{\mathrm{e}}$ siècle, on a assisté à un double phénomène d'appropriation: dans le champ des arts plastiques modernistes, le "primitivisme» procède par emprunt de formes issues d'autres cultures, remettant ainsi en cause les traditions classiques; parallèlement, des mouvements tels que l'anthropophagisme récupèrent des références et des modèles esthétiques occidentaux et déconstruisent à travers une logique carnavalesque les stéréotypes portant sur les civilisations dénuées de technologie. Cette dynamique de réappropriation qui est exacerbée par les agencements hétéroclites du postmodernisme reflète une neutralisation progressive des distinctions culturelles. Le travail filmique de Dominique Gonzalez-Foerster emprunte ses références au nouveau cinéma d'auteur asiatique, principalement diffusé dans le cadre de festivals internationaux, et à des structures narratives telles que le Nouveau Roman; certains plans remettent en scène des clichés visuels associés à l'exotisme, abordant ainsi de manière transversale la question $\mathrm{du}$ tropicalisme. Cette dernière peut parfois même y apparaître comme instrumentalisée, à travers une expérience décontextualisée qui n'engage qu'indirectement les aspects politiques des constructions identitaires.

Dans l'entretien avec Hans Ulrich Obrist, le travail de Dominique Gonzalez-Foerster est symptomatiquement envisagé à partir d'une définition synthétique du tropicalisme, en recourant à des termes tels que «environnement», "milieu organique puissant» ou encore "hyper-réceptivité non-verbale»8. Dominique Gonzalez-Foerster conçoit les œuvres d'art comme des environnements qui déplacent l'expérience traditionnelle de l'installation: l'espace d'exposition est envisagé comme un ter-
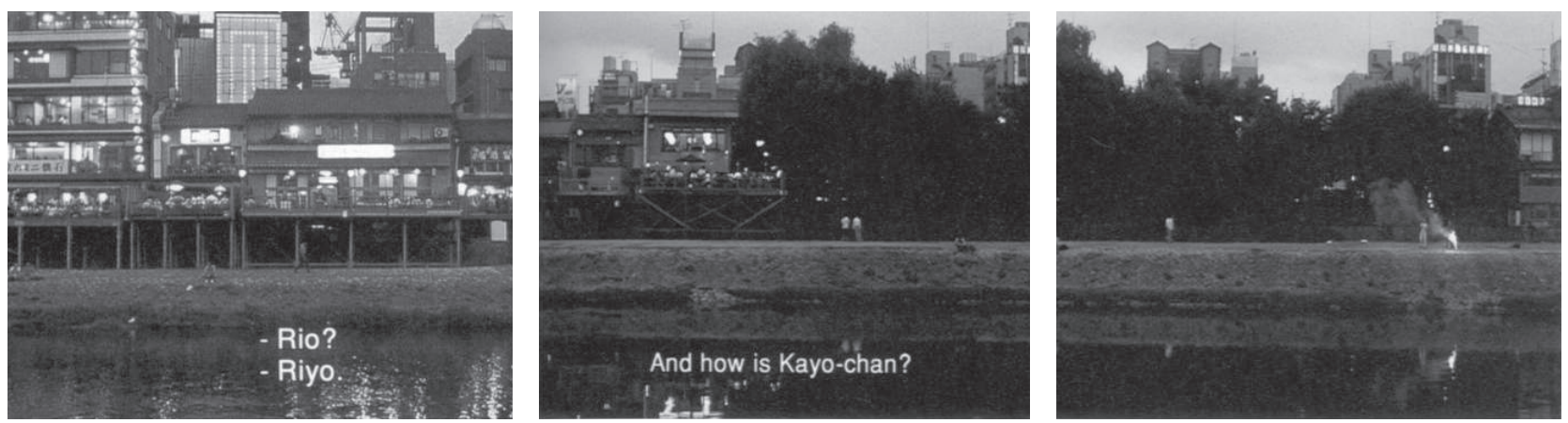
ritoire intermédiaire, réel et virtuel, qui engage le corps et la mémoire du spectateur. De son point de vue, l'art contemporain a la capacité d'induire un sentiment «super-signifiant» 9 , l'artiste rapportant cet «état d'hyper-réceptivité non-verbale» à la création d'un "espace potentiel de jeu» (tel que Donald Winnicott l'a analysé dans Jeu et réalité10). L'expérience de lecture proposée au spectateur, en raréfiant le langage verbal, neutralise l'espace et les spécificités culturelles: un point de vue global ne peut plus être reconstitué.

\section{Micro-événements et perspectives d'ensemble}

La tension entre le centre et la périphérie est également inscrite dans la logique du travail de Dominique Gonzalez-Foerster 11. L'artiste tourne des séquences filmiques qui peuvent être rejouées différemment selon leur contexte et leur format de présentation. Ainsi, des plans qui apparaissent dans Riyo (tourné à Kyoto et projeté en 2001 aussi bien au Festival de Cannes que dans un espace d'exposition à Francfort) rappellent Kyoto (1998), placé en ouverture du DVD produit par Anna Sanders Films. Le travelling le long d'une berge où se succèdent des immeubles fait l'objet d'une réinterprétation dont la différence réside dans la conception de la bande son et l'introduction d'une dimension narrative. L'articulation des courts métrages sur le support DVD génère un parcours où la circulation, à l'instar de l'espace d'exposition, permet différentes lectures; les chapitres constituent également des pièces autonomes, et leur ensemble un long métrage. Cette déambulation redouble l'expérience physique de l'espace urbain avec un effet de désorientation de nos repères spatio-temporels.

La structure même des courts métrages de Dominique GonzalezFoerster reproduit cette logique de la discontinuité, disloquant toute appréhension unitaire par la prégnance des détails. La logique fragmentaire
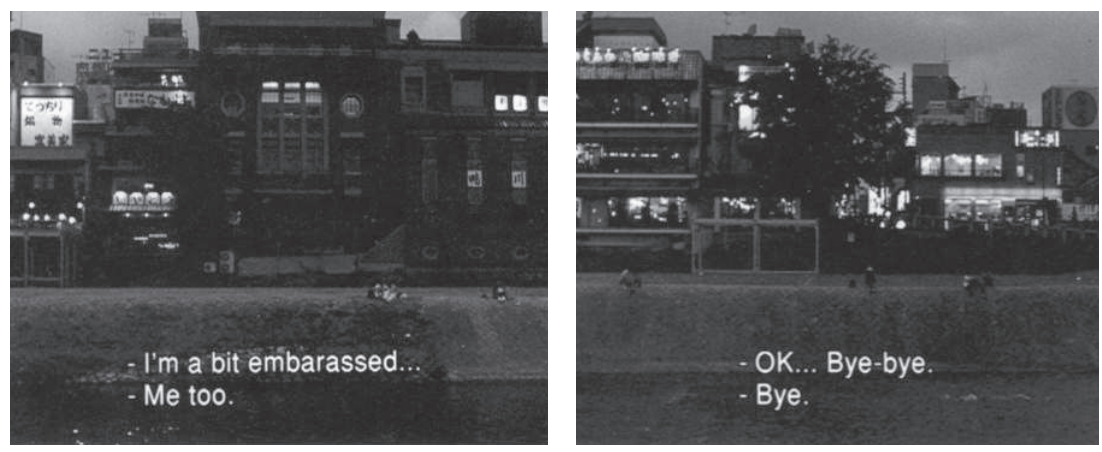

9 /d., p. 309.

10 Donald W. Winnicott, Playing and Reality, Tavistock, Londres, 1971 [Jeu et réalité. L'espace potentiel, Gallimard, Paris, 1975, traduit par Claude Monod et Jean-Bertrand Pontalis].

11 Nous ne prenons en compte dans cet article que le travail de Dominique Gonzalez-Foerster à partir d'images animées, excluant a fortiori la dynamique intermédiale et collective d'un certain nombre de ses projets artistiques (qu'il s'agisse de collaborations avec des artistes - Pierre Huyghe, Philippe Parreno, Ange Leccia, Martial Galfione -, avec des musiciens - Jay Jay Johansson, Christophe van Huffel, Alain Bashung - ou encore avec des architectes - Philippe Rahm, Ole Scheeren). Nous écartons également les films qu'elle a réalisés avec d'autres artistes, tels que İle de Beauté (1996, avec Ange Leccia), ce premier long métrage incarnant néanmoins le régime esthétique de l'errance et de la flânerie caractéristique de l'univers cinématographique de Dominique Gonzalez-Foerster.

Riyo (1999) ou Kyoto (1998) de Dominique Gonzalez-Foerster

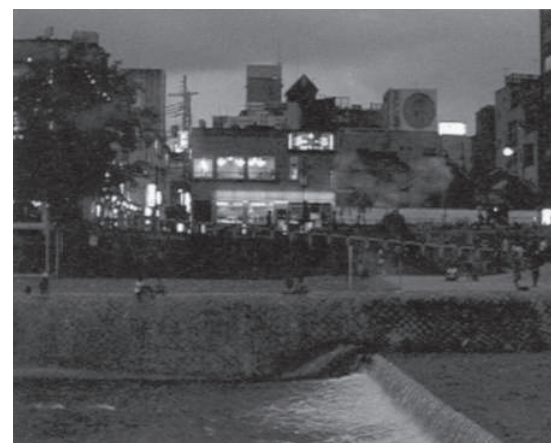


12 Daniel Birnbaum fait référence aux réflexions du poète américain du XIXe siècle Gerard Manley Hopkins qui s'est approprié le concept d'haeccéité, tel qu'il a été articulé dans le contexte de la philosophie scolastique. Principe d'individuation et d'existence, l'haeccéité repose sur un élément actuel qui constitue la subtance du sujet ou de la chose. Hopkins ressaisit cette notion à travers les catégories d'"inspect" (inscape) et d'«intension" (instress): l'inspect d'une chose, c'est sa forme, c'est-à-dire ce qui la constitue immédiatement et globalement comme une unité singulière; l'intension d'une chose, c'est la dynamique ou la concentration propre à la chose, ce qui la fonde dans son incomparable singularité; l'intension imprime ainsi un mouvement au schéma statique de l'inspect (sur ces points, voir Gerard Manley Hopkins, De l'origine de la beauté, Editions Comp'act, Seyssel, 1989, traduit par JeanPierre Audigier et René Gallet).

13 Daniel Birnbaum, "Rien", Chronologie, Les presses du réel/JRP Ringier, Dijon/Zurich, 2007, traduit de l'anglais par Simon Baril, p. 90.

14 Daniel Birnbaum (op. cit., p. 94) cite un passage de L'empire des signes de Roland Barthes (Albert Skira/Flammarion, Paris, 1970, p. 10): "L'écriture est en somme, à sa manière, un satori: le satori (l'événement Zen) est un séisme plus ou moins fort (nullement solennel) qui fait vaciller la connaissance, le sujet: il opère un vide de parole.".

15 Jacques Kerouac, Satori in Paris, Grove Press, New York, 1966.

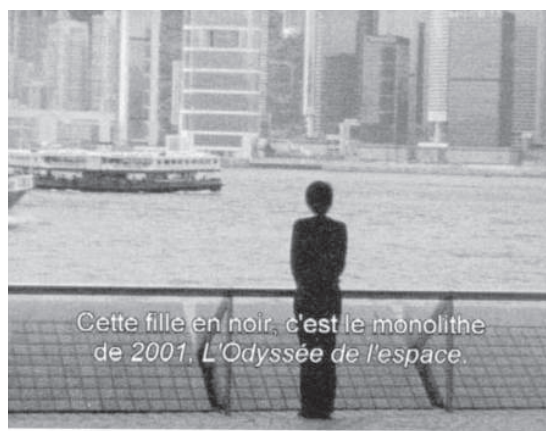

à l'œuvre dans le travail de l'artiste déconstruit la possibilité d'une lecture hiérarchisée: l'hypertrophie du détail induit un trouble de la vision, un décentrement du point de vue et une indétermination du sens. A travers le prisme de l'haeccéité12, Daniel Birnbaum définit le travail de Dominique Gonzalez-Foerster comme "des événements - des vagues ou des blocs de sensation - qui ne peuvent être intégrés dans des structures narratives plus larges» $\mathbf{1 3}$. Il met ainsi en valeur «l'absence stratégique de signification" qui est au cœur de la démarche de Dominique Gonzalez-Foerster, en convoquant l'expérience du satori tel que l'entendait Roland Barthes dans L'empire des signes $\mathbf{1 4}$. La forme du haïku, sur laquelle nous reviendrons, est ici définitoire: l'œuvre est dépouillée, articulée autour de micro-événements qui font saillie. De manière plus informelle, nous pourrions tout aussi bien renvoyer à l'expérience du satori telle que le mouvement de la Beat Generation l'a comprise, notamment Jacques Kerouac dans Satori in Paris $\mathbf{1 5}$ : un incident ou un détail signifiant, éveillant chez le sujet percevant des sensations kinesthésiques et un processus d'association d'idées, produit un effet d'étrangeté qui peut être lié à une impression de déjà-vu. Suivant un processus analogue à celui de la mémorisation, les détails perdent tout rapport de subordination: la prédominance d'un élément par rapport aux autres devient dès lors subjective. Les morceaux disparates s'agencent de façon improductive: les degrés de l'échelle de taille se dissolvent dans l'informe, générant l'atmosphère mélancolique caractéristique de l'univers filmique de Dominique Gonzalez-Foerster.

\section{Réfractions du cinéma dans l'espace public}

Les mouvements d'aller-retour entre le détail et la vue d'ensemble, les préoccupations quotidiennes et les configurations globales, caractérisent l'écriture cinématographique de Dominique Gonzalez-Foerster. Les
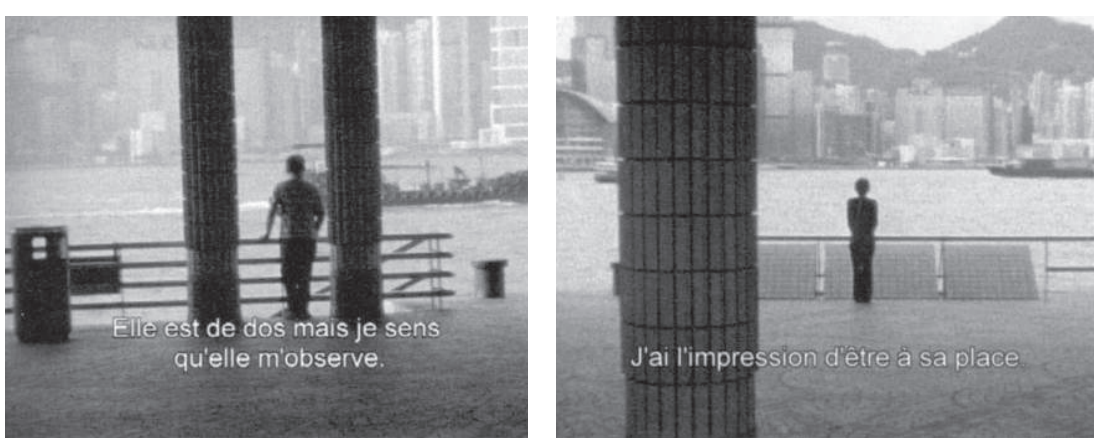
pièces qu'elle recueille dans le DVD Parc Central s'inscrivent dans le contexte de lieux publics, qu'il s'agisse de villes (Kyoto, Taipei, Buenos Aires, Hong Kong, Brasilia, Paris, Shangai, Rio de Janeiro) ou d'environnements naturels. Ceux-ci sont médiatisés par le souvenir de plans de films qui mettent en scène une ambiance annonciatrice d'un point culminant, d'un climax. En visionnant Parc Central, le spectateur fait l'expérience d'une interrelation entre le collectif et l'individuel, l'universel et le particulier, l'impression globale et le détail insignifiant, suivant un principe de réversibilité.

En un sens, Dominique Gonzalez-Foerster conçoit le cinéma comme une déambulation au sein d'un environnement où le naturel et l'artefact se confrontent, constituant ainsi un texte filmique condensé. Taipei (2000) s'apparente à une errance déterminée par la mémoire du dernier plan-séquence de Vive l'amour (Tsai-Ming Liang, Taïwan, 1994), expérience cinématographique qu'elle tente de reconstituer tout en constatant l'impossibilité de sa répétition. En ce sens, Taipei participe à une entreprise d'anthropophagie culturelle inversée, où il s'agit de "cannibaliser» les référents du cinéma taïwanais. L'origine de cette situation est différée, laissant dans l'indétermination la prééminence du modèle et la nature des références. La bande vidéo de Dominique Gonzalez-Foerster problématise la question de possibles particularismes culturels. A quels publics les films de Tsai-Ming Liang s'adressent-ils prioritairement? Comment le cinéaste se réapproprie-t-il des modèles isssus du cinéma d'art et d'essai européen et négocie-t-il son rapport vis-à-vis du nouveau cinéma taïwainais, dont l'essor est régulièrement associé à In Our Time (Yi Chang, I-Chen Ko, Te-chen Tao et Edward Yang, 1982)?

Dominique Gonzalez-Foerster rapporte le souvenir de ce plan à une scène primitive qui aurait impulsé son désir de cinéma. Mais ce n'est qu'à travers les sous-titres, détachés de toute parole proférée, que l'on peut

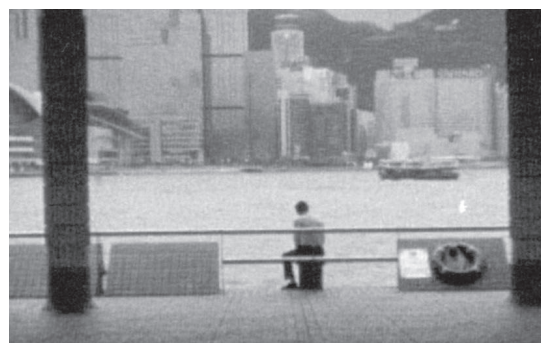

La nuit, c'est complètement diffèrent
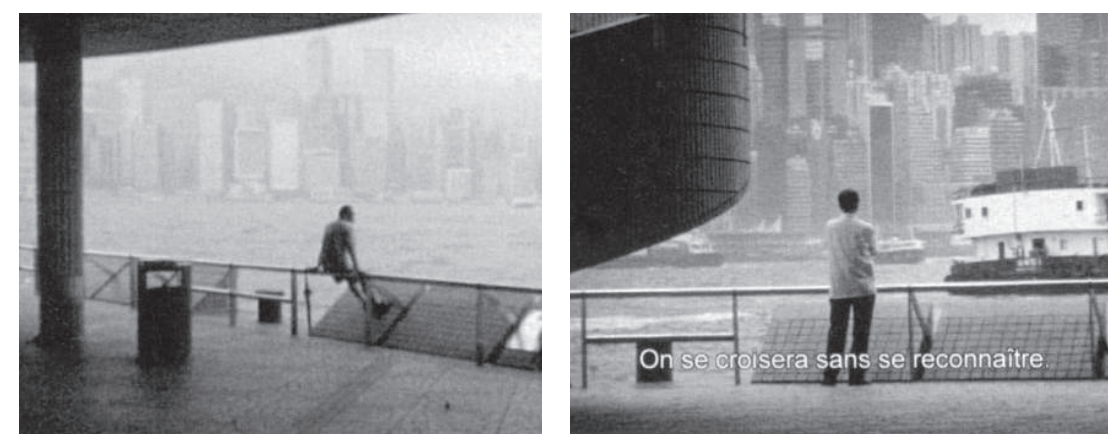

Central (2001) ou Hong Kong (2000) de Dominique Gonzalez-Foerster 

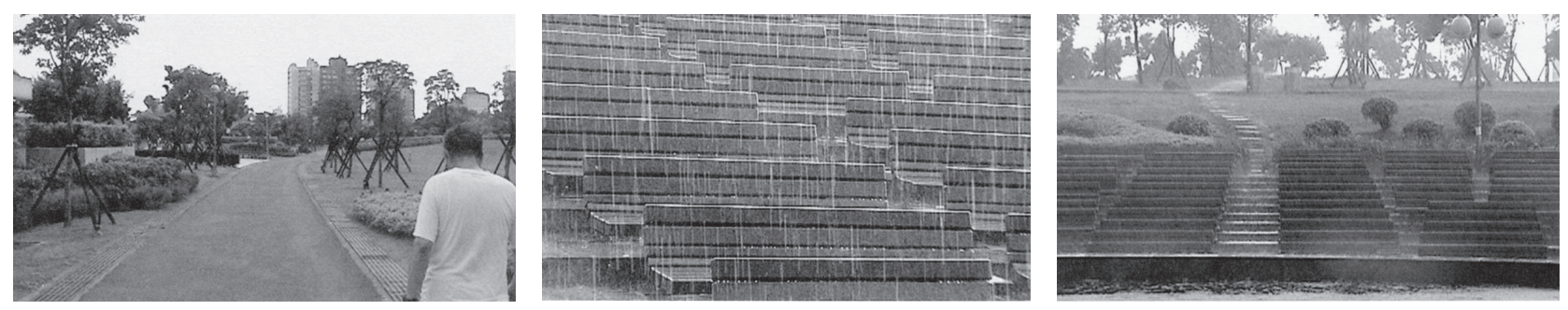

Atomic Park (2003) ou Taipei (2000) de Dominique Gonzalez-Foerster

induire ce propos. Au niveau de l'image, la reprise du finale du film de Tsai-Ming Liang (d'une durée de dix minutes qui sert d'étalon à la relecture opérée par Dominique Gonzalez-Foerster) est plutôt déceptive: les bancs et la scène du théâtre sont désormais vides ; à l'esthétique du planséquence longuement tenu se substituent des images vidéo instables, multipliant les zooms, un montage heurté et d'ultimes plans gelés qui se succèdent, interrompant le flux de l'enregistrement et soulignant l'irréalité de la séquence morcelée. Cette opposition entre low et high tech, entre souvenirs de vacances tournés par un amateur et film d'artiste, est assumée par la plasticienne, qui thématise dans sa pièce une inversion entre le dehors et le dedans, l'extérieur et l'intérieur: la pluie battante supplée désormais les pleurs de la protagoniste de Vive l'amour, effondrée sur un banc. L'intérêt de Taipei repose plus particulièrement sur l'ambivalence entretenue entre l'exacerbation d'une émotion et les mécanismes de secondarisation liés aux souvenirs reconstruits, qui engage à nouveau la question du collectif et de l'individuel. La mémoire propre, difficilement médiatisable, est ainsi articulée à travers les sous-titres :

«Dans les films de Tsai Ming-Liang, il pleut aussi beaucoup. C'est tropical. Toute cette pluie et toutes ces larmes. Ces larmes qui m'ont donné envie de faire des films. Il fallait que je voie ce parc ‘en vrai), que je retrouve la scène du film.»

La pluie, désignée comme un indice de tropicalité, est investie comme une expérience du temps mort et de l'attente : elle contraint Dominique Gonzalez-Foerster à s'immobiliser avec sa caméra sur la scène du théâtre; le spectacle devient dès lors le dispositif architectural où pourrait prendre place le public. L'artiste y aperçoit un plan de la ville qu'elle a laissé derrière elle, renvoyant ainsi à la perte de repères spatiaux suscités par l'orage et par analogie à une désorientation temporelle induite par le souvenir d'un plan-séquence qui efface la distinction entre fiction et réalité. La lecture muette des sous-titres induit également cette perméabilité des rôles, générant un effet de contamination où le spectateur s'identifie au mouvement du flux de conscience de l'énonciatrice. La 

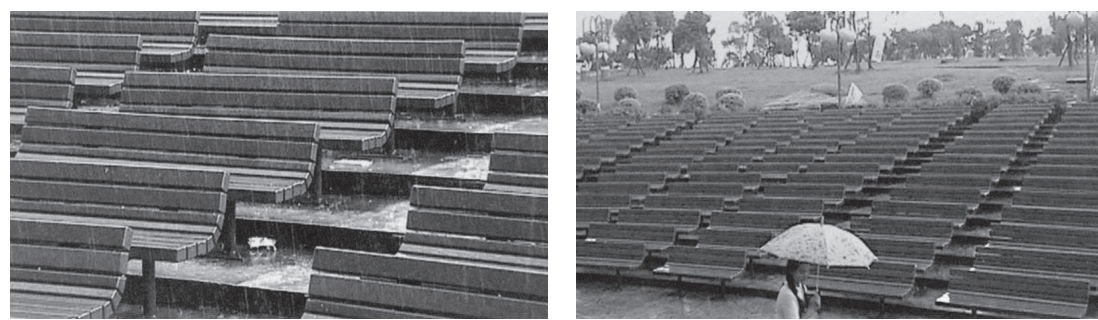

bande vidéo de Dominique Gonzalez-Foerster met bien en évidence le principe que les formalistes russes théorisaient, à savoir la traduction verbale qu'opère le spectateur lors du visionnement d'un film $\mathbf{1 6}$.

\section{Le paysage comme décor de cinéma, voire comme personnage}

L'un des projets définitoires d'Anna Sanders Films, comme on peut le lire dans la déclaration d'intention du collectif, consiste à dresser le portrait de lieux :

"[...] ce projet s'est voulu comme un moyen d'inventer - par le film - des moments de paysage... à travers le monde: faire naître à l'Histoire une plage brésilienne, le Pamir à la vérité des images... la jungle tropicale à l'évanouissement du temps. [...] Anna Sanders Films rêve de faire partager au spectateur sa liberté dans la perception des durées et des temporalités. $\mathbf{1 7}$

Selon cette perspective, le film s'annonce d'emblée comme l'expérience d'un voyage virtuel pour le spectateur. Ce déplacement mental est encore accentué dans le DVD Parc Central 18 édité par Anna Sanders; non seulement la plupart des titres sont composés de noms de villes, mais encore l'invitation au voyage figure sur la couverture du DVD:

«De Kyoto à Rio, en passant par Buenos Aires, Brasilia et Paris ce sont des parcs, des plages, des déserts, des moments urbains; des espaces traversés à pied avec une caméra. Un ensemble de séquences inédites filmées entre 1998 et 2003 et structurées comme un ‘concept album `. Autant de ‘morceaux > de paysages à regarder et à écouter avec la musique originale d'Adanowsky, Xavier Boussiron, Christophe van Huffel pour découvrir progressivement pourquoi on voyage, pourquoi on fait des films...»

Les paysages composés par des structures architecturales et des territoires indéterminés tels que la plage ou le désert, sont traversés comme des scènes de théâtre, voire des décors de cinéma. Ainsi, l'expérience du lieu est médiatisée par une culture cinématographique. Dans les courts métrages Brasilia (1998) et White Sands (2003), la figure du désert et ses

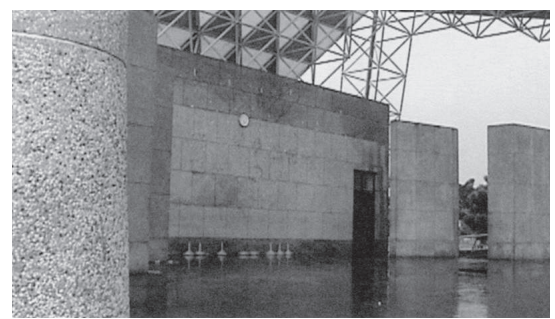

16 Sur la théorie du monologue intérieur au cinéma, voir Boris Eikhenbaum, "Problèmes de ciné-stylistique ", dans François Albera (éd.), Les Formalistes russes et le cinéma, Nathan, Paris, 1996, pp. 37-72.

17 "A propos d'Anna Sanders", complément au DVD de Dominique Gonzalez-Foerster Parc Central.

18 Le jeu de mot du titre de ce "long métrage" numérique manifeste une résistance à toute localisation assignable: le parc est central, globalisé; il n'est nullement question du lieu-dit Central Parc. 
résonances entropiques convoquent des souvenirs de films tels que Zabriskie Point (Michelangelo Antonioni, Etats-Unis/Italie, 1970), La cicatrice intérieure (Philippe Garrel, France, 1970) ou encore La région centrale (Michael Snow, Canada, 1971). Dans Brasilia, Dominique GonzalezFoerster convoque la mémoire d'un film de Philippe de Broca (L’homme de Rio, France, 1964) qui impliquait déjà le complexe architectural de Brasilia, dessiné par l'urbaniste Lúcio Costa, avec des bâtiments d'Oscar Niemeyer. En sous-titres défile le commentaire suivant de l'artiste:

«[...] J'avais vu des photos et des plans de cette ville symétrique des années 1960, en forme d'avion ou d'oiseau. Les gens traversent cette surface comme une scène immense. C'est Belmondo dans L'homme de Rio, en train de courir dans la ville en construction. L'idée c'était de faire une sorte de bande-annonce pour une ville différente, avec un centre vide, désert, plage ou parc. Une plate-forme centrale qui se traverse comme un film.»

La ville est perçue à travers les mouvements des véhicules et des personnes qui traversent l'espace, Dominique Gonzalez-Foerster suivant du regard leur déplacement; au contraire, les plans architecturaux sont statiques, à travers un cadrage large. L'opposition entre le minéral et l'organique que nous évoquions en ouverture de cet article est ainsi réinscrite formellement. Une tension est perceptible entre l'immobilisme des structures architectoniques et les déplacements qui animent le plan. Les mouvements de la caméra DV, tenue à la main, se superposent aux déplacements perçus dans le champ. Dominique Gonzalez-Foerster met constamment en avant les terrains en friche contre lesquels se découpent les bâtiments situés à l'arrière-plan, conférant un sentiment de contraste entre l'informe et la géométrie qui structure l'espace, entre le centre et la périphérie. De façon paradoxale, le terrain vague constitue le centre-ville, inversant ainsi les rapports de hiérarchie entre le cœur et les marges. Le film développe le rapport ambivalent entre l'organicité et le formalisme, déjà présent dans le plan urbanistique de Brasilia, qui a incité l'artiste à se rendre sur les lieux qu'elle ne connaissait que par l'intermédiaire de photographies. Le film se clôt sur un élément anecdotique: un avion est lancé dans le champ, motif synecdotique qui perturbe les rapports d'échelle entre la forme emblématique de la ville et ce jouet insignifiant. L'irruption de cet avion dans un décor qui rappelle les films de science-fiction intervient sur le mode de la surprise et de l'instantanéité, simulant la logique d'une bande-annonce.

Si Brasilia est une ville reconnaissable par ses bâtiments caractéristiques, White Sands est un site indéterminé (identifié par aucun nom); le noir et blanc rend ambiguë cette plage qui évoque par instants un 
paysage enneigé. La matérialité de l'esthétique filmique, perçue à travers les bandes d'amorce et les défauts du grain de la pellicule $16 \mathrm{~mm}$, trame l'espace d'un lieu désertique. L'architecture des cabines de ce qui apparait comme une aire de pique-nique ne se rapporte à la mémoire d'aucun site familier. Pourtant, une similitude dans l'approche des lieux relie ces deux courts métrages: à savoir une impression de proximité dans la perception d'un environnement dont les effets de perspective sont mis à plat. Il en résulte une difficulté à différencier les instants saillants d'un flux continu, une vision en gros plan d'une vue d'ensemble. Sur la bande-son, des riffs de guitare destructurés amplifient cette impression d'entropie.

Cette incapacité à pouvoir situer un point de vue constant, ce flottement dans la perception se retrouvent dans Rio de Janeiro (2000), dont la représentation figure un paysage mixte, une plage bordée de constructions urbaines. Des pavements en motifs de vagues reproduisent le mouvement de flux et de reflux de la mer, ainsi que la déambulation des passants; la modification de l'échelle des plans générée par les zooms avant et arrière souligne cette dynamique de va-et-vient. Par ailleurs, le son des ressacs succède à une chanson exotique sur la bande-son. La focalisation de ce plan-séquence, pris depuis une chambre d'hôtel avec un puissant téléobjectif, permet des modulations entre le détail et la vue d'ensemble. Ce dispositif optique évoque la description minutieuse que fait Raymond Roussel d'une photographie de plage enchassée dans un porte-plume dans $L a$ vue ${ }^{19}$, poème écrit en alexandrin qui met en exergue et anime les moindres détails d'un objet anodin tout en confondant la représentation et ce qui la fonde.

Dans Hong Kong (2000), le décor appelle à nouveau la métaphore, les carrelages d'un embarcadère de ferry étant comparés à une salle de bain démesurée, perturbant les rapports de proportions. L'indétermination de l'espace est surdéterminée par la transition fluide entre les éléments bâtis et le chantier en construction; inversement, la berge à perte de vue est délimitée par les bords du cadre. Tournant cette fois avec une caméra super 8, Dominique Gonzalez-Foerster restitue à nouveau un moment d'attente dans une ville étrangère, structurant un espace visuel et sonore. L'embarcadère appelle le souvenir d'un film asiatique, Chungking Express (Wong Kar-Wai, Hong-Kong, 1994). Le familier et l'inhabituel se croisent dans un lieu dont l'artiste ne maîtrise pas la langue, matière sonore qui suspend le sens pour offrir une relecture du réel. Le voyage s'appréhende comme une circulation et une transition vers des espaces fictionnels, passage possible vers un ailleurs mental. La transparence de la pluie et l'immatérialité des jeux de lumière génèrent un
19 Raymond Roussel, La Vue, Jean-Jaques Pauvert, Paris, 1963 [1904]. 
20 Ezra Pound, "In a Station of the Metro" [1913], Early Writings. Poems and Prose, Penguin Books, Londres, 2005, p. 82 ("Dans une station de métro: L'apparition de ces visages dans la foule; / Pétales sur une branche mouillée, noire.").

21 Voir l'analyse que Pound propose de son poème dans "Vorticism" [1914], Early Writings. Poems and Prose, op. cit., pp. 278-291. écran, une surface de projection potentielle qui transfigure cette jetée. Les immeubles à l'arrière-plan se voient ainsi dénaturés, apparaissant comme un décor factice, un environnement sculptural. A nouveau, les sous-titres établissent des liens entre le cinéma, le voyage et les villes: "Les liens entre les films et les villes, les films et les voyages. Les villes en langue étrangère. Le cinéma comme une langue étrangère.» L'expérience ainsi proposée au spectateur serait celle d'une ambivalence entre le lieu commun et une perception renouvelée, voire altérée. Ces motifs, oscillant entre un effet de carte postale et une composition picturale, posent la question du rapport entre un langage universel et motivé d'une part, immédiat et stratifié par la culture d’autre part.

\section{Un langage d'exploration}

Buenos Aires (2003) se déploie le temps d'une chanson, empruntant le format caractéristique du clip vidéo. Au centre-ville de Buenos Aires, intervient une situation incongrue: des papiers sont jetés des fenêtres des immeubles; ils jonchent progressivement la rue. L'événément est cadré et monté de sorte à évoquer le motif de la pluie, avec un rythme qui s'accélère progressivement. Le flottement d'un mouvement saisi au vol et des voitures au sol déstabilise l'espace quadrillé des immeubles. La tension entre les papiers en apesanteur et la rigidité des constructions remet en cause la logique de la gravité. L'ambivalence entre la légèreté et la densité caractéristique de l'univers cinématographique de Dominique Gonzalez-Foerster se cristallise autour de ce motif allégorique. Le modèle de la poésie visuelle, remontant à la structure du haïku et explorée dans les années 1910-1920 par l'écriture imagiste et vorticiste, constitue peut-être l'ultime horizon de référence des courts métrages de Dominique Gonzalez-Foerster. Dans un poème intitulé «Dans une station de métro", l'écrivain d'avant-garde devenu classique Ezra Pound transpose dans un contexte urbain un haiku de Moritake:

"In a Station of the Metro:

The apparition of these faces in the crowd;

Petals on a wet, black bough.» 20

Pound confronte la métaphore naturelle du haïku de Moritake (les pétales tombés d'une branche et un papillon) à un milieu urbain (les visages de la foule dans le métro). Dans un manifeste portant sur le mouvement vorticiste, Pound définit la poésie comme un "langage de l'exploration", en se référant aux modèles du haïku japonais et de la poésie chinoise. Dans ce contexte, il propose la catégorie du "poème d'une seule image", qu'il définit comme "une forme de super-position, c'est-à-dire qu'une idée en chevauche une autre» $\mathbf{2 1}$. Pound retrace le 
mode de composition de son poème: la reconfiguration kaléidoscopique par la mémoire d'une scène urbaine lui laisse une forte impression qu'il cherche à transposer par l'écriture, mais il bute contre les facilités rhétoriques de la métaphore; insatisfait du résultat, il élague son poème jusqu'à le réduire à deux vers. Ce qu'il vise à travers cette méthode de composition par résorption et cristallisation, c'est à une abolition de la distinction entre la subjectivité et l'objectivité, entre l'intériorisation et l'extériorisation de la conscience: "Dans un poème de cette espèce, on essaye d'enregistrer l'instant précis où une chose objective et extérieure se transforme, ou se propulse en une chose intérieure et subjective.»22

Ce modèle caractérise la démarche de Dominique Gonzalez-Foerster: dans le cas singulier de Buenos Aires, l'artiste transpose à son tour, bien que probablement involontairement, ce haiku dans le contexte urbain d'une métropole argentine. Figure de l'écart, le détail déplace les limites qui séparent des contextes éloignés; par ailleurs, le contour des choses se désagrège, tout en impliquant un processus de perception qui ouvre à une conscience réflexive. Le détail met en lumière les enjeux d'un instant prégnant, d'un moment décisif: le regard est arrêté, submergé par la dislocation de toute saisie unitaire du réel d'une part et par la densité de sensations qui laissent sans voix d'autre part. Le spectateur est conduit à creuser cet écart entre l'ordre fragmentaire et un horizon plus large. L'excès du visuel, qui peut être relié à la notion de "figurabilité » $\mathbf{2 3}$, sature l'espace de la représentation, ce qui conduit le spectateur à lui attribuer un contexte en traçant ses propres liens et associations. Comme le souligne Daniel Arasse, le détail est facteur de "rapprochements incongrus et presque contradictoires. Il ouvre des chemins de traverse dans les catégories [...] et décourage toute velléité d’un savoir 〈complet 〉.» $\mathbf{2 4}$
22 ld., p. 286.

23 Sur le concept de figurabilité, voir JeanFrançois Lyotard, Discours, figure, Klincksieck, Paris, 1971.

24 Daniel Arasse, Le détail. Pour une histoire rapprochée de la peinture, Flammarion, Paris, 1996 , p. 17. 